Pacific

Journal of

Mathematics

NONDEGENERACY OF COVERINGS OF MINIMAL TORI AND KLEIN BOTTLES IN RIEMANNIAN MANIFOLDS

JoHn DOUGLAS MOORE

Volume $230 \quad$ No. 2

April 2007 


\title{
NONDEGENERACY OF COVERINGS OF MINIMAL TORI AND KLEIN BOTTLES IN RIEMANNIAN MANIFOLDS
}

\author{
John Douglas MoORE
}

\begin{abstract}
We say that a parametrized minimal torus or Klein bottle in an ambient Riemannian manifold is Morse nondegenerate if it lies on a nondegenerate critical submanifold which is also an orbit for the group of isometries of the flat metric of total area one. We show that for a generic choice of a Riemannian metric on a compact manifold of dimension at least four, unbranched multiple covers of prime minimal tori or Klein bottles are Morse nondegenerate. A similar result holds for harmonic tori and Klein bottles. The proofs require a modification of techniques of Bott for studying iterations of smooth closed geodesics.
\end{abstract}

\section{Introduction}

Suppose that $\operatorname{Map}(\Sigma, M)$ is a suitable completion of the space of smooth maps $f: \Sigma \rightarrow M$ from a compact connected surface $\Sigma$ into a Riemannian manifold $M$ and that $\mathscr{T}$ is the space of marked conformal structures on $\Sigma$. (We complete with respect to a Sobolev norm strong enough so that $\operatorname{Map}(\Sigma, M)$ is a smooth infinitedimensional Banach manifold and there is a continuous inclusion $\operatorname{Map}(\Sigma, M) \subset$ $C^{0}(\Sigma, M)$ inducing an isomorphism on all homotopy groups.) A parametrized minimal surface can then be regarded as a critical point for the energy function

$$
E: \operatorname{Map}(\Sigma, M) \times \mathscr{T} \rightarrow \mathbb{R} \quad \text { defined by } \quad E(f, \omega)=\frac{1}{2} \int_{\Sigma}|d f|^{2} d A,
$$

where $|d f|$ and $d A$ are calculated with respect to some Riemannian metric on $\Sigma$ which lies within the conformal class $\omega \in \mathscr{T}$. A nonconstant parametrized minimal surface $f: \Sigma \rightarrow M$ is prime if it is not a nontrivial cover (possibly branched) of a parametrized minimal surface $f_{0}: \Sigma_{0} \rightarrow M$ of lower energy, $\Sigma_{0}$ being allowed to be nonorientable. In [Moore 2006] we proved a bumpy metric theorem which states that when a compact manifold $M$ of dimension at least four is given a generic Riemannian metric, all prime parametrized minimal surfaces $f: \Sigma \rightarrow M$ are free of

MSC2000: primary 53C40, 58E12; secondary 58D15, $58 \mathrm{E} 05$.

Keywords: minimal surfaces, harmonic surfaces, bumpy metrics, generic Riemannian metrics. 
branch points and are as Morse nondegenerate (as critical points for $E$ ) as allowed by the group of conformal automorphisms of $\Sigma$.

To be more precise about what we mean by Morse nondegenerate, we first recall that when $\Sigma$ is the sphere (or the projective plane), $E$ is invariant under an action of the six-dimensional Lie group $G=P S L(2, \mathbb{C})$ by linear fractional transformations on the range, while if $\Sigma$ is the torus (or the Klein bottle), $E$ is invariant under an action of the two-dimensional group $G=S^{1} \times S^{1}$. Thus nonconstant minimal spheres or tori must lie on orbits of critical points of dimension six or two, respectively. If $\Sigma$ (or its double cover) has genus at least two, we let $G$ denote the trivial group.

Definition. Let $F: M \rightarrow \mathbb{R}$ be a $C^{2}$ function on a smooth manifold $\mu$ which is modeled on a Hilbert or Banach space. A nondegenerate critical submanifold of $M$ is a finite-dimensional submanifold $N \subset \mathcal{M}$ such that every $f \in N$ is a critical point for $F$, and

$$
f \in N \quad \Rightarrow \quad T_{f} N=\left\{X \in T_{f} \mathcal{M}: d^{2} F(f)(X, Y)=0 \text { for all } Y \in T_{f} \mathcal{M}\right\} .
$$

Here $d^{2} F$ is the Hessian of $F$ at the critical point, and elements $X \in T_{f} \mathcal{M}$ which satisfy the condition on the right-hand side of (2) are called Jacobi fields.

The notion of a nondegenerate critical submanifold is due to Bott [1982] and plays a large role in the Morse theory of closed geodesics.

Definition. We say that a parametrized minimal surface $f: \Sigma \rightarrow M$ is Morse nondegenerate if either $f$ is a Morse nondegenerate critical point for $E$ in the usual sense of Morse theory, or $f$ lies on a $G$-orbit which is a nondegenerate critical submanifold for $E$.

With these definitions in place, the bumpy metric theorem of [Moore 2006] can be restated: if $M$ has dimension at least three, then for generic choice of Riemannian metric on $M$, all prime parametrized minimal surfaces are Morse nondegenerate. This can be regarded as an analog of Abraham's [1970] bumpy metric theorem for smooth closed geodesics, which asserts that for generic metrics on $M$, all smooth closed geodesics lie on nondegenerate critical submanifolds of $\operatorname{Map}\left(S^{1}, M\right)$, each an orbit for the $S^{1}$ action. However, an important difference is that Abraham's bumpy metric theorem applies to all closed geodesics, prime or not.

In analogy with the theory of smooth closed geodesics, one might hope that unbranched covers of tori and Klein bottles also lie on nondegenerate critical submanifolds for generic metrics, and this is in fact the case:

Theorem 1. Suppose that $M$ is a compact smooth manifold of dimension at least four with Riemannian metric $g_{0}$. 
(1) If $f_{0}: \Sigma_{0} \rightarrow M$ is a Morse nondegenerate prime minimal torus or Klein bottle with no branch points, then for a generic choice of Riemannian metric on $M$ near $g_{0}$, all minimal tori and Klein bottles which cover $f_{0}$ are also Morse nondegenerate.

(2) if $f_{0}: \Sigma \rightarrow M$ is a nonoriented Morse nondegenerate prime minimal surface of any genus with no branch points, then for a generic choice of Riemannian metric on $M$ near $g_{0}$, the oriented double cover of $f_{0}$ is also Morse nondegenerate.

We adopt the convention that the genus of a connected nonorientable surface is the genus of its orientable double cover. From this theorem and the Main Theorem of [Moore 2006], it follows that for generic choice of Riemannian metric on $M$, all parametrized minimal tori are free of branch points and Morse nondegenerate, except for branched covers of minimal two-spheres, which are of course forced to have branch points. Although it can be shown that branched covers of spheres by tori lie on smooth submanifolds of dimension $2 d+2$, where $d$ is the degree, there is no reason to suspect that these submanifolds should be Morse nondegenerate for generic choice of Riemannian metric.

Thus if $M$ is not simply connected, all parametrized minimal tori in one of the nontrivial components of $\operatorname{Map}\left(T^{2}, M\right) \times \mathscr{T}$ lie on nondegenerate critical submanifolds of dimension two for generic choice of Riemannian metric on $M$. The motivation behind Theorem 1 is that it serves as part of the foundation necessary for a study of Morse theory for parametrized minimal surfaces via perturbation, using the $\alpha$-energy of Sacks and Uhlenbeck [1981; 1982].

Following [McDuff and Salamon 2004], we say that a conformal harmonic map $f: \Sigma \rightarrow M$ from a compact surface $\Sigma$ is somewhere injective if there exists at least one point $p \in \Sigma$ such that $f^{-1}(f(p))=\{p\}$. It then follows from unique continuation theorems that the set of points $p$ for which $f^{-1}(f(p))=\{p\}$ is open and dense. Note that if $f$ is an imbedding, it is somewhere injective, and indeed, $f^{-1}(f(p))=\{p\}$ for every $p \in \Sigma$. On the other hand, nontrivial branched covers are not somewhere injective. It follows from the theory of branched immersions developed by Gulliver, Osserman and Royden [Gulliver et al. 1973], or directly from Lemma 4.1 of [Moore 2006], that prime parametrized minimal surfaces are always somewhere injective, and the proof of the Main Theorem of the latter article uses this fact.

To prove that multiple covers are nondegenerate for generic choice of metric, we extend part of Bott's theory of iterated closed geodesics [Bott 1956]. In the argument, we use the following fact that can be proven using the implicit function theorem. If a prime parametrized minimal surface $f_{0}$ is Morse nondegenerate for a metric $g_{0}$ on $M$, then there is a unique $G$-orbit of Morse nondegenerate minimal 
surfaces with the same topology near $f_{0}$ for any metric near $g_{0}$. (If $\Sigma$ is the twosphere or projective plane, it is convenient to replace the group $\operatorname{PSL}(2, \mathbb{C})$ by its compact subgroup $S O(3)$, by fixing the center of mass.) Thus it suffices to consider perturbations in the metric $g_{0}$ which have the same one-jet as $g_{0}$ along $f_{0}$, and therefore preserve minimality of $f_{0}$. A corresponding fact was used in the proof given in [Klingenberg and Takens 1972; Klingenberg 1978] for the bumpy metric theorem for closed geodesics, and indeed our argument can be thought of as an extension of that proof from one to two dimensions.

A similar bumpy metric theorem (Theorem 2) will be formulated for harmonic tori and Klein bottles in Section 5 and proved in Section 6. We will see that in the case of nonconformal harmonic maps, we must deal with a new phenomenon, the possibility of fold points.

\section{Preliminaries}

We first recall some basic concepts from the theory of harmonic and minimal surfaces in Riemannian manifolds, further details being found in [Micallef and Moore 1988] and [Moore 2006]. If we fix the conformal structure $\omega \in \mathcal{T}$, the energy reduces to the $\omega$-energy

$$
E_{\omega}: \operatorname{Map}(\Sigma, M) \rightarrow \mathbb{R}, \quad \text { defined by } \quad E_{\omega}(f)=E(f, \omega),
$$

the critical points of which are called $\omega$-harmonic maps. To determine the equation for harmonic maps, we take the first derivative of $E_{\omega}$, obtaining

$$
d E_{\omega}(f)(X)=\int_{\Sigma}\left\langle F_{\omega}(f, g), X\right\rangle d A, \quad \text { for } X \in \Gamma\left(f^{*} T M\right),
$$

and set $F_{\omega}(f, g)=0$, where $F_{\omega}(\cdot, g)$ is the Euler-Lagrange operator. If one chooses local conformal coordinates $\left(x_{1}, x_{2}\right)$ on $\Sigma$, and lets $\lambda^{2}$ denote the conformal factor in the metric, so that the area element is given by $d A=\lambda^{2} d x_{1} d x_{2}$, the equation one obtains is

$$
\frac{D}{\partial x_{1}}\left(\frac{\partial f}{\partial x_{1}}\right)+\frac{D}{\partial x_{2}}\left(\frac{\partial f}{\partial x_{2}}\right)=0, \quad \text { where } \quad \frac{\partial f}{\partial x_{a}}=f_{*}\left(\frac{\partial}{\partial x_{a}}\right)
$$

is regarded as a section of the bundle $f^{*} T M$ over $\Sigma$ for $a=1,2$, and $D$ denotes the pullback of the Levi-Civita connection of $M$ to $f^{*} T M$.

Differentiating $E_{\omega}$ once again gives us the Hessian at a critical point,

$$
d^{2} E_{\omega}(f)(X, Y)=\int_{\Sigma}\left\langle D_{1} F_{\omega}(f, g)(X), Y\right\rangle d A=\int_{\Sigma}\left\langle L_{\omega}(X), Y\right\rangle d A,
$$

for $X, Y \in \Gamma\left(f^{*} T M\right)$. Here $D_{1} F_{\omega}$ denotes the derivative with respect to the variable $f \in \operatorname{Map}(\Sigma, M)$ and $L_{\omega}$ is the Jacobi operator for $E_{\omega}$, which acts on sections 
of the pullback $f^{*} T M$ of the tangent bundle to $M$, or equivalently, elements of the tangent space to $\operatorname{Map}(\Sigma, M)$ at $f$. The Jacobi equation is $L_{\omega}(X)=0$ when $X \in \Gamma\left(f^{*} T M\right)$, and solutions to this equation are called Jacobi fields for the function $E_{\omega}$. A well-known calculation shows that

$$
d^{2} E_{\omega}(f)(X, X)=\int_{\Sigma}\left(\|D X\|^{2}-\langle\mathscr{K}(X), X\rangle\right) d A,
$$

where in terms of the complex parameter $z=x_{1}+i x_{2}$ on $\Sigma$,

and

$$
\|D X\|^{2}=\frac{1}{\lambda^{2}}\left(\left\|\frac{D X}{\partial x_{1}}\right\|^{2}+\left\|\frac{D X}{\partial x_{2}}\right\|^{2}\right)
$$

$$
\langle\mathscr{K}(X), X\rangle=\frac{1}{\lambda^{2}}\left(\left\langle R\left(X, \frac{\partial f}{\partial x_{1}}\right) \frac{\partial f}{\partial x_{1}}, X\right\rangle+\left\langle R\left(X, \frac{\partial f}{\partial x_{2}}\right) \frac{\partial f}{\partial x_{2}}, X\right\rangle\right),
$$

$R$ being the Riemann-Christoffel curvature tensor of $M$.

A harmonic map $f$ is said to be conformal if it satisfies the conditions

$$
\left\langle\frac{\partial f}{\partial x_{1}}, \frac{\partial f}{\partial x_{1}}\right\rangle=\left\langle\frac{\partial f}{\partial x_{2}}, \frac{\partial f}{\partial x_{2}}\right\rangle \text { and }\left\langle\frac{\partial f}{\partial x_{1}}, \frac{\partial f}{\partial x_{2}}\right\rangle=0 .
$$

Parametrized minimal surfaces, the critical points of the two-variable energy $E$, are exactly the conformal harmonic maps.

The only possible singularities of parametrized minimal surfaces are branch points. These are most easily described in terms of a local complex parameter $z=x_{1}+i x_{2}$ (for the oriented double cover if $\Sigma$ is nonorientable). If we let

$$
\frac{\partial f}{\partial z}=\frac{1}{2}\left(\frac{\partial f}{\partial x_{1}}+i \frac{\partial f}{\partial x_{2}}\right)
$$

a section of the complexified tangent bundle $f^{*} T M \otimes \mathbb{C}$, a point $p \in \Sigma$ is a branch point if $(\partial f / \partial z)(p)=0$. If the coordinate $z$ is centered at $p$, we can then write $(\partial f / \partial z)=z^{v} g$, where $g(p)$ is nonzero, and $v$ is the branching order of $f$ at $p$.

If $\Sigma$ is oriented, the locally defined sections $(\partial f / \partial z)$ generate a line bundle $\boldsymbol{L}$ contained in $f^{*} T M \otimes \mathbb{C}$, which can be extended smoothly to the branch points. If $\Sigma$ is not orientable, the line bundle $\boldsymbol{L}$ can be defined over the oriented double cover. In either case, the real and imaginary parts of sections of $\boldsymbol{L}$ determine a two-dimensional subbundle $\left(f^{*} T M\right)^{\top}$ of $f^{*} T M$, which possesses an orthogonal complement $\left(f^{*} T M\right)^{\perp}$, yielding a direct sum decomposition

$$
f^{*} T M=\left(f^{*} T M\right)^{\top} \oplus\left(f^{*} T M\right)^{\perp} .
$$

Thus if $f$ is a conformal harmonic map, we can speak of tangent and normal sections of $f^{*} T M$ even if $f$ has branch points. 
Just as we did for $E_{\omega}$, we can calculate the first and second derivatives of the two-variable energy function $E: \operatorname{Map}(\Sigma, M) \times \mathscr{T} \rightarrow \mathbb{R}$. These derivatives are calculated in [Moore 2006, §5], and are essential for studying conformal harmonic maps with branch points.

However, the Main Theorem of [Moore 2006] says that for generic choice of metric all parametrized minimal surfaces are free of branch points, and hence immersions. On the space of immersions $f: \Sigma \rightarrow M$, it is simpler to consider the area function $A(f)$, defined by

$$
A(f)=\int\left|\frac{\partial f}{\partial x} \wedge \frac{\partial f}{\partial y}\right| d x d y .
$$

There is a classical formula for second variation of area under normal variations when $f: \Sigma \rightarrow M$ is a minimal immersion, which is presented in [Simons 1968, Theorem 3.2.2], [Lawson 1980, Theorem 32], and many other places. It states that if $X$ is a section of the normal bundle $\Gamma\left(\left(f^{*} T M\right)^{\perp}\right)$,

$$
\begin{array}{r}
d^{2} A(f)(X, X)=\int_{\Sigma}\left(\left\|(D X)^{\perp}\right\|^{2}-\langle\mathscr{B}(X)+\mathscr{K}(X), X\rangle\right) d A, \\
\text { where }\langle\mathscr{B}(X), X\rangle=\left\|(D X)^{\top}\right\|^{2},
\end{array}
$$

and $(\cdot)^{\perp}$ and $(\cdot)^{\top}$ denote projections into the tangent and normal spaces respectively. It is this second variation formula that we use in the proof of the Theorem. Just as in the case of $E_{\omega}$, we obtain a formally self-adjoint Jacobi operator

$$
L^{\perp}: \Gamma\left(\left(f^{*} T M\right)^{\perp}\right) \rightarrow \Gamma\left(\left(f^{*} T M\right)^{\perp}\right),
$$

from the second variation formula, such that

$$
d^{2} A(f)\left(X_{1}, X_{2}\right)=\int_{\Sigma}\left\langle L^{\perp}\left(X_{1}\right), X_{2}\right\rangle d A, \quad \text { for } \quad X_{1}, X_{2} \in \Gamma\left(\left(f^{*} T M\right)^{\perp}\right),
$$

which we call the normal Jacobi operator. We call a solution to $L^{\perp}(X)=0$ a normal Jacobi field.

It can be proved that in normal directions (7) gives the same result as second variation of the two-variable energy $E$ when the conformal structure is constrained to move in such a way that conformality of $f$ is preserved.

\section{Tori covering tori}

When the Riemann surface $\Sigma$ is a torus, the Teichmüller space $\mathscr{T}$ is the upper half plane

$$
\mathbb{H}=\{\omega=u+i v \in \mathbb{C}: v>0\},
$$

the point $\omega=u+i v$ corresponding to the conformal class of the torus $\mathbb{C} / \Lambda$, where $\Lambda$ is the lattice in $\mathbb{C}$ generated by 1 and $\omega$. After a change of basis we can arrange 
that a given element $\omega \in \mathscr{T}$ lies in the fundamental domain

$$
D=\left\{u+i v \in \mathbb{C}:-\frac{1}{2} \leq u \leq \frac{1}{2}, u^{2}+v^{2} \geq 1\right\}
$$

for the action of the group $S L(2, \mathbb{Z})$, the action having kernel $\{ \pm 1\}$. The moduli space $\mathscr{R}$ for the torus is the quotient of the upper half plane by the $S L(2, \mathbb{Z})$-action, and is obtained from $D$ by identifying points on the boundary. It is well-known that $\mathscr{R}$ is diffeomorphic to the space $\mathbb{C}$ of complex numbers.

The complex torus corresponding to a given $\omega \in \mathcal{T}$ can be regarded as the quotient of $\mathbb{C}$ by the abelian subgroup generated by $d$ and $\omega d$, where $d$ is any positive real number, or alternatively, this torus is obtained from a fundamental parallelogram spanned by $d$ and $\omega d$ by identifying opposite sides. If $\omega=u+i v$, we take $d=1 / \sqrt{v}$. Then the fundamental parallelogram has area one, and can be regarded as the image of the unit square $\left\{\left(t_{1}, t_{2}\right) \in \mathbb{R}^{2}: 0 \leq t_{i} \leq 1\right\}$ under the linear transformation

$$
\left(\begin{array}{l}
t_{1} \\
t_{2}
\end{array}\right) \mapsto\left(\begin{array}{l}
x \\
y
\end{array}\right)=\frac{1}{\sqrt{v}}\left(\begin{array}{ll}
1 & u \\
0 & v
\end{array}\right)\left(\begin{array}{l}
t_{1} \\
t_{2}
\end{array}\right),
$$

where $z=x+i y$ is the usual complex coordinate on $\mathbb{C}$. A straightforward calculation gives a formula for the energy:

$$
\begin{aligned}
E(f, \omega) & =\frac{1}{2} \int_{P}\left(\left|\frac{\partial f}{\partial x}\right|^{2}+\left|\frac{\partial f}{\partial y}\right|^{2}\right) d x d y \\
& =\frac{1}{2} \int_{P}\left(v\left|\frac{\partial f}{\partial t_{1}}\right|^{2}+\frac{1}{v}\left|\frac{\partial f}{\partial t_{2}}-u \frac{\partial f}{\partial t_{1}}\right|^{2}\right) d t_{1} d t_{2},
\end{aligned}
$$

$P$ denoting the unit square in the coordinates $\left(t_{1}, t_{2}\right)$. Both coordinate systems $\left(t_{1}, t_{2}\right)$ and $(x, y)$ on $\mathbb{C}$ are useful, the first respecting the product structure on the torus, the second being conformal.

Let $P_{k, l}$ be the parallelogram described by $0 \leq t_{1} \leq k$ and $0 \leq t_{2} \leq l$, whenever $k$ and $l$ are positive integers. If $f_{0}: T^{2} \rightarrow M$ is a prime minimal torus with conformal structure $\omega, f_{0}$ lifts to a map $\tilde{f}_{0}: \mathbb{C} \rightarrow M$ which can then be restricted to $P_{k, l}$. By identifying opposite edges of $P_{k, l}$ we obtain a torus $T^{2}$ with the conformal structure $(l / k) \omega$ and $\tilde{f}_{0}$ induces a conformal harmonic mapping $f_{1}$ from the new torus into $M$ which covers $f_{0}$ with multiplicity $k l$ and has fundamental parallelogram $P_{k, l}$.

We now carry through several constructions motivated by Bott's treatment of multiple covers of closed geodesics [1956]. Suppose as above that $f_{0}: T^{2} \rightarrow M$ is a prime minimal torus with lift $\tilde{f}_{0}: \mathbb{C} \rightarrow M$. Let $\left(\tilde{f}_{0}^{*} T M\right)^{\perp}$ denote the normal bundle to the immersion $\tilde{f}_{0}$ and if $z$ and $w$ are elements of $S^{1} \subset \mathbb{C}$, let

$\mathscr{V}_{(k, l),(z, w)}=\left\{\right.$ smooth sections $X$ of $\left(\tilde{f}_{0}^{*} T M\right)^{\perp} \otimes \mathbb{C}$ such that

$$
\left.X\left(t_{1}+k, t_{2}\right)=z X\left(t_{1}, t_{2}\right), X\left(t_{1}, t_{2}+l\right)=w X\left(t_{1}, t_{2}\right)\right\} .
$$


Define an Hermitian inner product

$$
\langle\cdot, \cdot\rangle_{k, l}: \mathscr{V}_{(k, l),(z, w)} \times \mathscr{V}_{(k, l),(z, w)} \rightarrow \mathbb{C}
$$

by

$$
\langle X, \bar{Y}\rangle_{k, l}=\int_{P_{k, l}}\left(\left\langle\frac{D X}{\partial x}, \frac{D \bar{Y}}{\partial x}\right\rangle+\left\langle\frac{D X}{\partial y}, \frac{D \bar{Y}}{\partial y}\right\rangle+\langle X, \bar{Y}\rangle\right) d x d y,
$$

where $D$ denotes the covariant derivative in the normal bundle defined by the LeviCivita connection on $M$ and the bar denotes conjugation. Note that elements of $\mathscr{V}_{(k, l),(1,1)}$ project to sections of $\left(f_{1}^{*} T M\right)^{\perp} \otimes \mathbb{C}$, where $f_{1}$ is the torus with fundamental parallelogram $P_{k, l}$.

Lemma 1. The inclusion

$$
\sum_{z^{k}=1} \sum_{w^{l}=1} \mathscr{V}_{(1,1),(z, w)} \subset \mathscr{V}_{(k, l),(1,1)},
$$

the sum being taken over all $k$-th and $l$-th roots of unity, is an isomorphism.

Proof. Since the inclusion is clearly injective, it suffices to show that it is surjective. If $X \in \mathscr{V}_{(k, l),(1,1)}$ and $z$ and $w$ are $k$-th and $l$-th roots of unity, we let

$$
X_{z, w}\left(t_{1}, t_{2}\right)=\frac{1}{k l} \sum_{a=0}^{k-1} \sum_{b=0}^{l-1} z^{-a} w^{-b} X\left(t_{1}+a, t_{2}+b\right) .
$$

Then

$$
\begin{aligned}
X_{z, w}\left(t_{1}+1, t_{2}\right) & =\frac{1}{k l} \sum_{a=0}^{k-1} \sum_{b=0}^{l-1} z^{-a} w^{-b} X\left(t_{1}+(a+1), t_{2}+b\right) \\
& =\frac{z}{k l} \sum_{a=0}^{k-1} \sum_{b=0}^{l-1} z^{-(a+1)} w^{-b} X\left(t_{1}+(a+1), t_{2}+b\right)=z X_{z, w}\left(t_{1}, t_{2}\right),
\end{aligned}
$$

and by a similar calculation,

$$
X_{z, w}\left(t_{1}, t_{2}+1\right)=w X_{z, w}\left(t_{1}, t_{2}\right),
$$

so $X_{z, w} \in \mathscr{V}_{(1,1),(z, w)}$. Moreover, an easy calculation shows that

$$
X=\sum_{z^{k}=1} \sum_{w^{l}=1} X_{z, w}
$$

so the inclusion (8) is indeed an isomorphism.

We next define an index form

$$
I_{k, l}(\cdot, \cdot): \mathscr{V}_{(k, l),(z, w)} \times \mathscr{V}_{(k, l),(z, w)} \rightarrow \mathbb{C}
$$

by 


$$
I_{k, l}(X, \bar{Y})=\int_{P_{k, l}}(\langle D X, D \bar{Y}\rangle-\langle\mathscr{B}(X)+\mathscr{K}(X), \bar{Y}\rangle) d A
$$

where $D$ is the connection in the normal bundle and $\mathscr{B}$ and $\mathscr{K}$ are the endomorphisms of the normal bundle appearing in the Simons formula (7). Recall that the operator $\mathscr{B}$ depends on the second fundamental form of $\tilde{f}_{0}$, while $\mathscr{K}$ is linear in the components of the curvature tensor. Both $\mathscr{B}$ and $\mathscr{K}$ are periodic since they are lifts of operators from $f_{0}$.

We can integrate by parts in (9), obtaining

$$
I_{k, l}(X, \bar{Y})=-\int_{P_{k, l}}(\langle\Delta X+\mathscr{B}(X)+\mathscr{K}(X), \bar{Y}\rangle) d A=\int_{P_{k, l}}\left\langle L^{\perp}(X) \bar{Y}\right\rangle d A
$$

where $\Delta$ is the Laplace operator defined by the normal connection and $L^{\perp}$ is the normal Jacobi operator, $X$ being a normal Jacobi field if and only if it satisfies the equation

$$
L^{\perp}(X)=-\Delta X-\mathscr{B}(X)-\mathscr{K}(X)=0 .
$$

We divide the parallelogram $P_{k, l}$ into $k l$ parallelograms $\left\{P^{a, b}\right\}$, where $a$ and $b$ are integers ranging from 1 to $k$ and 1 to $l$ respectively, the parallelogram $P^{a, b}$ being defined by the conditions $a-1 \leq t_{1} \leq a$ and $b-1 \leq t_{2} \leq b$. Note that if $X \in \mathscr{V}_{(1,1),\left(z_{1}, w_{1}\right)}$ and $Y \in \mathscr{V}_{(1,1),\left(z_{2}, w_{2}\right)}$, where $z_{1}$ and $z_{2}$ are $k$-th roots of unity and $w_{1}$ and $w_{2}$ are $l$-th roots of unity, and $X$ and $Y$ are extended to elements of $\mathscr{V}_{(k, l),(1,1)}$, the index form on the extensions is given by

$$
\begin{aligned}
I_{k, l}(X, \bar{Y}) & =\sum_{a=1}^{k} \sum_{b=1}^{l} \int_{P^{a, b}}(\langle D X, D \bar{Y}\rangle-\langle\mathscr{B}(X)+\mathscr{K}(X), \bar{Y}\rangle) d A \\
& =\sum_{a=1}^{k} \sum_{b=1}^{l}\left(z_{1} \bar{z}_{2}\right)^{a}\left(w_{1} \bar{w}_{2}\right)^{b} \int_{P^{1,1}}(\langle D X, D \bar{Y}\rangle-\langle\mathscr{B}(X)+\mathscr{K}(X), \bar{Y}\rangle) d A .
\end{aligned}
$$

Thus we see that

$$
I_{k, l}(X, \bar{Y})= \begin{cases}k l I_{1,1}(X, \bar{Y}) & \text { if } z_{1}=z_{2} \text { and } w_{1}=w_{2}, \\ 0 & \text { if } z_{1} \neq z_{2} \text { or } w_{1} \neq w_{2}\end{cases}
$$

and hence the direct sum decomposition

$$
\sum_{z^{k}=1} \sum_{w^{l}=1} \mathscr{V}_{(1,1),(z, w)}
$$

is orthogonal with respect to the index form $I_{k, l}$. In particular, whenever $z$ and $w$ are roots of unity, the normal Jacobi operator $L^{\perp}$ defined by (10) restricts to an endomorphism

$$
L^{\perp}: \mathscr{V}_{(1,1),(z, w)} \rightarrow \mathscr{V}_{(1,1),(z, w)}
$$


Let $N(z, w)$ denote the nullity of the index form $I_{k, l}$ restricted to $\mathscr{V}_{(1,1),(z, w)}$,

$$
\begin{aligned}
N(z, w) & =\operatorname{dim}_{\mathbb{C}}\left\{X \in \mathscr{V}_{(1,1),(z, w)}: I_{k, l}(X, \bar{Y})=0 \text { for all } Y \in \mathscr{V}_{(1,1),(z, w)}\right\} \\
& =\operatorname{dim}_{\mathbb{C}}\left\{X \in \mathscr{V}_{(1,1),(z, w)}: L^{\perp}(X)=0\right\} .
\end{aligned}
$$

The preceding discussion proves the following lemma, analogous to a lemma of Bott [1956] which plays a key role in his analysis of the relationship between the index and nullity of a prime smooth closed geodesic and the index and nullity of its multiple covers:

Lemma 2. If $z_{1}$ and $w_{1}$ are primitive $k$-th and $l$-th roots of unity,

$$
\text { Nullity of } f_{1}=\sum_{a=1}^{k} \sum_{b=1}^{l} N\left(z_{1}^{a}, w_{1}^{b}\right) \text {. }
$$

We now turn to the proof of Theorem 1 in the case of tori covering tori. Our strategy is to perturb the metric in a neighborhood of the given Morse nondegenerate minimal surface $f_{0}: T^{2} \rightarrow M$ in such a way that $f_{0}$ is preserved, but the Jacobi equations are perturbed.

We construct a variation of the Riemannian metric on $M$ of a specific form. We choose a point $p \in T^{2}$ such that $f_{0}^{-1}\left(f_{0}(p)\right)=\{p\}$, and a neighborhood $U$ containing $p$ such that $f_{0}$ imbeds $U$ into some open set $V \subset M$. Arrange, moreover, that $V$ is the domain of local coordinates $\left(u_{1}, \ldots, u_{n}\right)$ such that $u_{i}\left(f_{0}(p)\right)=0$ and

(1) $f_{0}(U)$ is described by the equations $u_{3}=\cdots=u_{n}=0$,

(2) $u_{a} \circ f_{0}=x_{a}$ on $f_{0}(U)$, for $a=1,2$, where $x_{1}+i x_{2}$ is a conformal parameter on $U$, and

(3) the Riemannian metric $g$ on the ambient space takes the form $\sum g_{i j} d u_{i} d u_{j}$, such that when restricted to $f_{0}(\Sigma) \cap V, g_{i r}=\delta_{i r}$, for $1 \leq i \leq n$ and $3 \leq r \leq n$.

Such coordinates can be constructed using the exponential map restricted to the normal bundle of the surface $f_{0}(\Sigma) \cap V$ in $M$.

Following [Klingenberg 1978, proof of Proposition 3.3.7], we construct a variation $\dot{g}=\sum \dot{g}_{i j} d u_{i} d u_{j}$ of the metric on the ambient manifold $M$ such that

$$
\begin{gathered}
\dot{g}_{11}\left(u_{1}, \ldots u_{n}\right)=\dot{g}_{22}\left(u_{1}, \ldots u_{n}\right)=\sum_{r, s=3}^{n} u_{r} u_{s} \alpha_{r s}\left(u_{1}, \ldots, u_{n}\right), \\
\dot{g}_{i j}=0 \quad \text { if }(i, j) \neq(1,1) \text { or }(2,2) .
\end{gathered}
$$

Here the $\alpha_{r s}$ are smooth functions which vanish outside a small tubular neighborhood of $f_{0}\left(\Sigma_{0}\right)$. A straightforward calculation shows that the resulting changes in 
the Christoffel symbols

$$
\dot{\Gamma}_{k i j}=\frac{1}{2}\left(\frac{\partial \dot{g}_{i k}}{\partial u_{j}}+\frac{\partial \dot{g}_{j k}}{\partial u_{i}}-\frac{\partial \dot{g}_{i j}}{\partial u_{k}}\right)
$$

vanish except for

$$
\begin{aligned}
& \dot{\Gamma}_{r 11}=\dot{\Gamma}_{r 22}=-\sum_{s=3}^{n} u_{s} \alpha_{r s}, \\
& \dot{\Gamma}_{1 r 1}=\dot{\Gamma}_{11 r}=\dot{\Gamma}_{2 r 2}=\dot{\Gamma}_{22 r}=\sum_{s=3}^{n} u_{s} \alpha_{r s}, \quad \text { for } 3 \leq r \leq n .
\end{aligned}
$$

We want to consider the effect of such a variation on the operator $L^{\perp}$. Since the operator $\mathscr{B}$ (which is essentially the second fundamental form of $\left(\tilde{f}_{0}^{*} T M\right)^{\perp}$ in $\tilde{f}_{0}^{*} T M$ ) depends only on the Christoffel symbols along $f_{0}$ (where $u_{r}=0$ ), the variation of $\mathscr{B}$ is zero under the metric deformation. On the other hand, the variation of the operator $\mathscr{K}$ depends on the changes in curvature components, which are given by the formulae $\dot{R}_{l i j k}=\frac{\partial}{\partial u_{i}}\left(\dot{\Gamma}_{l j k}\right)-\frac{\partial}{\partial u_{j}}\left(\dot{\Gamma}_{l i k}\right)+\sum_{m} \dot{\Gamma}_{l i m} \Gamma_{j k}^{m}+\sum_{m} \Gamma_{l i m} \dot{\Gamma}_{j k}^{m}-\sum_{m} \dot{\Gamma}_{l j m} \Gamma_{i k}^{m}-\sum_{m} \Gamma_{l j m} \dot{\Gamma}_{i k}^{m}$. Along $f_{0}\left(\Sigma_{0}\right)$ all the $\dot{\Gamma}_{k i j}$ and $\dot{\Gamma}_{i j}^{k}$ must vanish, so along $f_{0}\left(\Sigma_{0}\right)$,

$$
\dot{R}_{1 r 1 s}=\dot{R}_{2 r 2 s}=\frac{\partial}{\partial u_{r}}\left(\dot{\Gamma}_{11 s}\right)-\frac{\partial}{\partial u_{1}}\left(\dot{\Gamma}_{1 r s}\right)=\alpha_{r s} .
$$

Thus the normal metric variation will result in the following variation of the endomorphism $\mathscr{K}$ :

$$
\dot{\mathscr{T}}\left(\sum_{r=3}^{n} h_{r} \frac{\partial}{\partial u_{r}}\right)=\sum_{r, s=3}^{n} \alpha_{r s} h_{s} \frac{\partial}{\partial u_{r}},
$$

where the $h_{r}$ can be arbitrary real-valued functions.

We let $\operatorname{Met}\left(M, f_{0}\right)$ denote the space of Riemannian metrics $g$ on $M$ such that the one-jet $j_{1}(g)$ of $g$ agrees with the one-jet $j_{1}\left(g_{0}\right)$ of $g_{0}$ at points of $f_{0}\left(\Sigma_{0}\right)$. This implies that $f_{0}: T^{2} \rightarrow M$ is a minimal torus for any $g \in \operatorname{Met}\left(M, f_{0}\right)$. Given any metric $g \in \operatorname{Met}\left(M, f_{0}\right)$, we have a corresponding normal Jacobi operator

$$
L_{g}^{\perp}: \Gamma\left(\left(f_{0}^{*} T M\right)^{\perp}\right) \rightarrow \Gamma\left(\left(f_{0}^{*} T M\right)^{\perp}\right), \quad L_{g}^{\perp}=-\Delta-\mathscr{B}-\mathscr{K}(g),
$$

the Laplace operator $\Delta$ and the second fundamental form endomorphism $\mathscr{B}$ being independent of the choice of $g \in \operatorname{Met}\left(M, f_{0}\right)$.

We now consider a given cover $f_{1}: T^{2} \rightarrow M$ of $f_{0}$. We suppose, as above, that $f_{0}$ has fundamental parallelogram $P$ while $f_{1}$ has fundamental parallelogram 
$P_{k, l}$. By Lemma 2, the contributions to the nullity of $f_{1}$ come from Jacobi fields in $N(z, w)$, as $z$ and $w$ range over the $k$-th and $l$-th roots of unity. We let

$$
E_{k}(z, w)=\text { completion of } \mathscr{V}_{(1,1),(z, w)} \text { with respect to the } L_{k}^{2} \text {-norm, }
$$

and define a map

$$
F: E_{k}(z, w) \times \operatorname{Met}\left(M, f_{0}\right)_{k}^{2} \rightarrow E_{k-2}(z, w) \quad \text { by } \quad F(X, g)=L_{g}^{\perp}(X) .
$$

Note that for each choice of $g, X \mapsto L_{g}^{\perp}(X)$ is a linear Fredholm map of Fredholm index zero.

We let $S E_{k}(z, w)=\left\{X \in E_{k}(z, w):\|X\|=1\right\}$, the fiber of a unit sphere bundle

$$
S E_{k}(z, w) \times \operatorname{Met}\left(M, f_{0}\right)_{k}^{2} \rightarrow \operatorname{Met}\left(M, f_{0}\right)_{k}^{2} .
$$

We claim that the subset

$$
\mathscr{S}=\left\{(X, g) \in S E_{k}(z, w) \times \operatorname{Met}\left(M, f_{0}\right)_{k}^{2}: L_{g}^{\perp}(X)=0\right\}
$$

is a smooth submanifold. Note that any $X$ with $\|X\|=1$ such that $L_{g}^{\perp}(X)=0$ must be nonzero on an open dense set and hence any unit-length element of $E_{k-2}(z, w)$ not in the image of $L_{g}^{\perp}$ must be nonzero on an open dense set. Thus it follows from (12) that any element of $E_{k-2}(z, w)$ not in the image of $L_{g}^{\perp}$ is of the form $D_{2} F(X, g)(\dot{g})$ for some metric variation $\dot{g} \in T_{g}\left(\operatorname{Met}\left(M, f_{0}\right)_{k}^{2}\right)$. Thus the restriction of $F$ to the total space of the sphere bundle is a submersion, and our claim follows immediately from the implicit function theorem.

Suppose now that

$$
\pi: S E_{k}(z, w) \times \operatorname{Met}\left(M, f_{0}\right)_{k}^{2} \rightarrow \operatorname{Met}\left(M, f_{0}\right)_{k}^{2}
$$

is the projection on the second factor. We claim that the restriction of $\pi$ to $\mathscr{Y}$,

$$
\pi: \mathscr{Y} \rightarrow \operatorname{Met}\left(M, f_{0}\right)_{k}^{2},
$$

is a Fredholm map of Fredholm index -1 . To see this, we note first that

$$
\begin{aligned}
T_{(X, g)} \mathscr{Y}=\left\{(Y, \dot{g}) \in E_{k}(z, w)\right. & \times T_{g} \operatorname{Met}\left(M, f_{0}\right)_{k}^{2} \\
& \text { such that } \left.L_{g}^{\perp}(Y)+D_{2} F(X, g)(\dot{g})=0,\langle X, Y\rangle=0\right\},
\end{aligned}
$$

where $\langle\cdot, \cdot\rangle$ is the $L_{k}^{2}$ inner product. Thus $(Y, \dot{g})$ lies in the kernel of $d \pi_{(X, g)}$ if and only if $\langle X, Y\rangle=0$ and $L_{g}^{\perp}(Y)=0$. Since $X$ is in the kernel of $L_{g}^{\perp}$ by definition of $\mathscr{Y}$, the dimension of the kernel of $d \pi_{(X, g)}$ is one less than the dimension of the kernel of the Jacobi operator $L_{g}^{\perp}$. 
We next investigate the cokernel, noting first that $\dot{g} \mapsto D_{2} F(X, g)(\dot{g})$ covers the cokernel of $L_{g}^{\perp}$. For $Y \in E_{k-2}(z, w)$, we define a continuous linear functional

$$
T(Y): T_{g} \operatorname{Met}\left(M, f_{0}\right)_{k}^{2} \rightarrow \mathbb{R} \quad \text { by } T(Y)(\dot{g})=\left\langle\left(D_{2} F\right)_{(X, g)}(\dot{g}), Y\right\rangle_{k-2},
$$

where $\langle\cdot, \cdot\rangle_{k-2}$ denotes the $L_{k-2}^{2}$ inner product. If $\dot{g}$ is in the range of $d \pi_{(X, g)}$, then $T(Y)(\dot{g})=0$ whenever $Y$ is perpendicular to the range of $L_{g}^{\perp}$. This shows that the codimension of the range of $d \pi_{(X, g)}$ is the dimension of the cokernel of $L_{g}^{\perp}$. Thus $\pi$ is indeed a Fredholm map of index

$$
\operatorname{dim}\left(\text { Kernel of } L_{g}^{\perp}\right)-1-\operatorname{dim}\left(\text { Cokernel of } L_{g}^{\perp}\right)=-1 .
$$

It therefore follows from the Sard-Smale theorem [Smale 1965] that for $g$ belonging to a countable intersection of open dense subsets of $\operatorname{Met}\left(M, f_{0}\right)_{k}^{2}$, there will be no solutions $X$ to $L_{g}^{\perp}(X)=0$ in $E_{k}(z, w)$. There are only a countable number of tori covering a given torus, and for each covering of type $(k, l)$ only $k l$ choices of roots of unity $z$ and $w$. Therefore for a countable intersection of open dense subsets of $\operatorname{Met}\left(M, f_{0}\right)_{k}^{2}$, there will no nonzero normal Jacobi fields for any torus covering a given nondegenerate minimal torus. This, together with the remarks at the end of the Introduction, proves Theorem 1 for tori covering tori.

\section{Nonorientable surfaces}

We next consider the modifications necessary to treat the case in which the prime minimal torus is replaced by a prime minimal Klein bottle $f_{0}: K^{2} \rightarrow M$.

A minimal Klein bottle will be double covered by a minimal torus with a flat metric of area one that is invariant under an orientation-reversing deck transformation. Such a deck transformation is a map $A_{s}: T^{2} \rightarrow T^{2}$ which is expressed in terms of appropriate standard coordinates $\left(t_{1}, t_{2}\right)$ on the torus as

$$
A_{s}\left(t_{1}, t_{2}\right)=\left(t_{1}+\frac{1}{2},-t_{2}-s\right)
$$

for $s \in S^{1}$, and consists of a translation composed with a reflection. One easily checks that this map satisfies the identity $A_{s}^{2}=1$. Recall that the Teichmüller space $\mathcal{T}$ for the torus is the upper half-plane $\mathbb{H}$, the point $\omega=u+i v \in \mathbb{H}$ corresponding to the torus $\mathbb{C} / \Lambda$, where $\Lambda$ is generated by 1 and $\omega$. In the case of a double cover of a Klein bottle, we arrange that the differential of $A_{s}$ fixes the generator corresponding to 1 in the fundamental parallelogram, and the differential must then take $\omega$ to $-\omega$. Since the differential of $A_{s}$ is an isometry for the flat metric, 1 and $\omega$ must be perpendicular, and the Teichmüller space of flat Klein bottles with total area one consists of the positive imaginary numbers $\omega=i v$ with $v>0$, the fixed point set of the involution

$$
A_{\star}: \mathbb{H} \rightarrow \mathbb{M}, \quad A_{\star}(u+i v)=-u+i v .
$$


(See [Wolf 1967, Proposition 2.5.8] for further discussion.)

As the space of maps of Klein bottles into $M$, we can take

$$
\operatorname{Map}\left(K^{2}, M\right)=\left\{f \in \operatorname{Map}\left(T^{2}, M\right): f \circ A_{s}=f \text { for some } s \in S^{1}\right\} .
$$

Minimal Klein bottles can then be regarded as critical points for the restricted twovariable function

$$
E: \operatorname{Map}\left(K^{2}, M\right) \times\left\{\omega \in \mathscr{T}: A_{\star}(\omega)=\omega\right\} \rightarrow \mathbb{R} .
$$

The energy is invariant under the action of $S^{1} \times S^{1}$ on $\operatorname{Map}\left(K^{2}, M\right)$ defined by

$$
f\left(t_{1}, t_{2}\right) \mapsto f\left(t_{1}+s_{1}, t_{2}+s_{2}\right), \quad \text { for } \quad\left(s_{1}, s_{2}\right) \in S^{1} \times S^{1} .
$$

In the subsequent discussion, we let $A=A_{s_{0}}$ for some choice of $s_{0} \in S^{1}$, thereby breaking part of the $S^{1} \times S^{1}$-symmetry.

Suppose now that $f: \Sigma \rightarrow M$ is an oriented double cover of a nonorientable minimal surface $f_{0}: \Sigma_{0} \rightarrow M$. The map $A$ induces an involution $A_{\star}$ on $f^{*} T M$, as well as on the space of sections of $f^{*} T M$, and both of these actions extend complex linearly to the complexifications. Moreover, these involutions preserve both the metric and the pullback of the Levi-Civita connection. If $\left.\boldsymbol{E}=\left(f^{*} T M\right)\right)^{\perp} \otimes \mathbb{C}$, the complexified normal bundle, the map $A_{\star}$ determines a direct sum decomposition $\Gamma(\boldsymbol{E})=\Gamma_{+}(\boldsymbol{E}) \oplus \Gamma_{-}(\boldsymbol{E})$, where

$$
\Gamma_{+}(\boldsymbol{E})=\left\{X \in \Gamma(\boldsymbol{E}): A_{\star}(X)=\bar{X}\right\}, \quad \Gamma_{-}(\boldsymbol{E})=\left\{X \in \Gamma(\boldsymbol{E}): A_{\star}(X)=-\bar{X}\right\} .
$$

The sections of $\Gamma_{+}(\boldsymbol{E})$ can be regarded as normal deformations of the nonorientable minimal surface $f_{0}: \Sigma \rightarrow M$ while the sections of $\Gamma_{-}(\boldsymbol{E})$ are deformation of $f$ that do not come from deformations of the underlying Klein bottle. The second variation formula (7) for area under normal variations applies immediately to sections of $\boldsymbol{E}$.

A key point is that the normal Jacobi operator $L^{\perp}$ must commute with $A_{\star}$, since $A_{\star}$ preserves the normal connection and the operators $\mathscr{B}$ and $\mathscr{K}$, and hence induces maps

$$
L^{\perp}: \Gamma_{+}(\boldsymbol{E}) \rightarrow \Gamma_{+}(\boldsymbol{E}) \quad \text { and } \quad L^{\perp}: \Gamma_{-}(\boldsymbol{E}) \rightarrow \Gamma_{-}(\boldsymbol{E}) .
$$

Since $f_{0}$ is nondegenerate, there are no normal Jacobi fields in $\Gamma_{+}(\boldsymbol{E})$, and the argument presented in Section 3 shows that for a generic choice of metric in $\operatorname{Met}\left(M, f_{0}\right)_{k}^{2}$, there will also be no normal Jacobi fields in $\Gamma_{-}(\boldsymbol{E})$. The argument from Section 3 also shows that there are no Jacobi fields in any torus covering $f$ and hence in any Klein bottle or torus covering $f_{0}$. This finishes the proof of the theorem for Klein bottles.

Finally, it remains only to establish the second statement in the Theorem, and this is a relatively straightforward modification of the previous argument. 
Suppose that $f: \Sigma \rightarrow M$ is an oriented double cover of a nonorientable minimal surface $f_{0}: \Sigma_{0} \rightarrow M$ of arbitrary genus, with $A$ being the sheet interchange map, so $f \circ A=f$. As in the special case of the Klein bottle, the map $A$ induces an involution $A_{\star}$ on the complexified normal bundle $\left.\boldsymbol{E}=\left(f^{*} T M\right)\right)^{\perp} \otimes \mathbb{C}$ of $f$, as well as on the space of sections of $\boldsymbol{E}$, and we have a direct sum decomposition (14). Just as before, there are no Jacobi fields in $\Gamma\left(\boldsymbol{E}_{+}\right)$since $f_{0}$ is assumed to be Morse nondegenerate, and the argument presented in Section 3 shows that for generic choice of metric in $\operatorname{Met}\left(M, f_{0}\right)_{k}^{2}$, there will also be no Jacobi fields in $\Gamma\left(\boldsymbol{E}_{-}\right)$. This finishes the proof of Theorem 1.

\section{Bumpy metrics for harmonic maps}

If $f$ is an $\omega$-harmonic map, the Hopf differential is the holomorphic quadratic differential

$$
\Omega_{f}=\left\langle\frac{\partial f}{\partial z}, \frac{\partial f}{\partial z}\right\rangle d z^{2},
$$

and it vanishes precisely when $f$ is conformal (that is, it satisfies (6)). Note that $\Omega_{f}$ automatically vanishes at branch points.

In the case where $\Sigma$ is the torus $T^{2}, \Omega_{f}=a d z^{2}$, where $a$ is a complex constant. If $f$ is not conformal, $a \neq 0$, and $f$ cannot have any branch points. On the other hand, if $a=0$, it follows from the Main Theorem of [Moore 2006] that $f$, now a parametrized minimal surface, has no branch points for generic choice of Riemannian metric on $M$, when $M$ has dimension at least four.

However, in contrast with minimal tori, nonconformal $\omega$-harmonic tori $f: T^{2} \rightarrow$ $M$ can have points at which the rank of $d f$ is one. This can happen in one of two ways: $f$ can be a torus parametrization of a smooth closed geodesic, or $f$ can have fold points.

Example. To see how the second case arises, we consider a degree zero harmonic map $f: T^{2} \rightarrow S^{2}$, where $S^{2}$ is given the standard Riemannian metric of constant curvature one, which has "fold points" along two circles parallel to the equator. To construct $f$, we first note that the metric on $S^{2} \subset \mathbb{R}^{3}$ with equation $x^{2}+y^{2}+z^{2}=1$ is expressed in spherical coordinates $(\phi, \theta)$, where $z=\cos \phi$ and $\theta$ is the standard angular coordinate in the $(x, y)$-plane, as

$$
d s^{2}=\left(\cos ^{2} \phi\right) d \theta^{2}+d \phi^{2}=\operatorname{sech}^{2} u\left(d \theta^{2}+d u^{2}\right),
$$

where $u$ and $\phi$ are related by the equation $\tanh (u / 2)=\tan (\phi / 2)$. In terms of the standard coordinates $\left(t_{1}, t_{2}\right)$ on $T^{2}$, we can define a mapping $f: T^{2} \rightarrow S^{2}$ by

$$
\theta\left(t_{1}, t_{2}\right)=t_{2}, \quad \phi\left(t_{1}, t_{2}\right)=\phi\left(t_{1}\right),
$$

where $\phi$ is a (nonconstant speed) parametrization of the geodesic $\theta=$ (constant). 
The circle $\phi=$ constant has curvature $\kappa=1 / \cos \phi$ and normal curvature $\kappa_{n}=1$. The equation $\kappa_{g}^{2}+\kappa_{n}^{2}=\kappa^{2}$, where $\kappa_{g}$ is the geodesic curvature, implies that $\kappa_{g}=$ $\pm \tan \phi$. Moreover, the curve is traversed with constant speed $\cos \phi$. Hence

$$
0=\frac{D}{\partial t_{1}}\left(\frac{\partial f}{\partial t_{1}}\right)+\frac{D}{\partial t_{2}}\left(\frac{\partial f}{\partial t_{2}}\right)=\frac{d^{2} \phi}{d t^{2}}+(\tan \phi)\left(\cos ^{2} \phi\right)=\frac{d^{2} \phi}{d t^{2}}+\frac{1}{2} \sin 2 \phi .
$$

Thus the differential equation we must solve to obtain a harmonic map (the pendulum equation except for constant factors) is equivalent to the first order system

$$
d \phi / d t=\psi, \quad d \psi / d t=\frac{1}{2} \sin 2 \phi .
$$

Eliminating $d t$ yields

$$
\frac{d \psi}{d \phi}=\frac{\frac{1}{2} \sin 2 \phi}{\psi}, \quad \text { which integrates to } \frac{1}{2} \psi^{2}+\frac{1}{2} \cos 2 \phi=\text { (constant). }
$$

For any constant strictly less than one-half we get solutions to the differential equations which yield harmonic maps for appropriate conformal structures on $T^{2}$. As the constant approaches one-half, the conformal structure approaches the boundary of Teichmüller space for the torus.

The antipodal map $A: S^{2} \rightarrow S^{2}$ induces an orientation reversing map $A: T^{2} \rightarrow T^{2}$ such that $f \circ A=A \circ f$. We can take the quotient in both domain and range, obtaining thereby a harmonic map from a Klein bottle into the real projective plane $\mathbb{R} P^{2}$, which has as its image a Möbius band.

Thus it is possible to construct four types of nonconstant harmonic tori without branch points which are not branched covers of minimal spheres, do not degenerate to geodesics, and do not cover harmonic tori of lower energy: immersed tori, double covers of immersed Klein bottles, harmonic cylindrical bands and double covers of harmonic Möbius bands.

The isotropy group $\Gamma$ of the $O(2) \times O(2)$-action on $\operatorname{Map}\left(T^{2}, M\right)$ is trivial for immersed tori and $\mathbb{Z}_{2}$ for harmonic cylindrical bands, the generator being a reflection in one of the two $O(2)$-factors. The isotropy group is $\mathbb{Z}_{2}$ for Klein bottles and $\mathbb{Z}_{2} \oplus \mathbb{Z}_{2}$ for harmonic Möbius bands.

We say that a harmonic surface $f: \Sigma \rightarrow M$ covers a harmonic surface $f_{0}: \Sigma_{0} \rightarrow$ $M$ if there is a conformal map $g: \Sigma \rightarrow \Sigma_{0}$ such that $f=f_{0} \circ g$.

Definition. An $\omega$-harmonic map $f: \Sigma \rightarrow M$ is prime if it is nonconstant and is not a cover (with possible branch and fold points) of an $\omega^{\prime}$-harmonic map $f_{0}: \Sigma_{0} \rightarrow M$ of lower energy, the surfaces $\Sigma$ and $\Sigma_{0}$ being allowed to be nonorientable.

Theorem 2. Suppose $M$ is a compact connected smooth manifold of dimension at least four with a generic choice of Riemannian metric. Then the nonconstant prime $\omega$-harmonic tori and Klein bottles are free of branch points. Moreover, either 
(1) they are parametrizations of smooth closed geodesics and lie on one-dimensional nondegenerate critical submanifolds, or

(2) they lie on two dimensional critical submanifolds, each an orbit for the action of $O(2) \times O(2)$ on $\operatorname{Map}\left(T^{2}, M\right)$.

The same holds for all $\omega$-harmonic tori and Klein bottles which are unbranched covers of $\omega^{\prime}$-harmonic tori and Klein bottles.

When the dimension of $M$ is three, a version of Theorem 2 holds for nonconformal harmonic maps.

In Theorem 2, we allow the possibility that the minimal surfaces may have fold points, points at which the fiber of the line bundle $\boldsymbol{L}$ coincides with its conjugate. It would be interesting to determine whether fold points exist in manifolds of dimension at least four with generic metrics.

In analogy with Theorem 1, the motivation behind Theorem 2 is that it is needed to provide part of the foundation for a partial parametrized Morse theory for $\omega$ harmonic maps, the conformal structure $\omega$ being the parameter.

\section{Proof of Theorem 2}

The proof of Theorem 2 is similar to that of Theorem 1, except that we use second variation of $E_{\omega}$ instead of second variation of $A$. Once one proves Theorem 2 for prime harmonic maps, the extension to unbranched covers is proven in exactly the same way as in Sections 3 and 4.

If $f$ is conformal, it follows from the Main Theorem of [Moore 2006] that $f$ is free of branch points. If $M$ is nonconformal, it cannot have branch points, as we already mentioned. Proposition 3.1 of the same paper shows that the theorem holds for those prime harmonic maps $f$ which are free of branch points and somewhere injective, that is satisfy the condition that $f^{-1}(f(p))=p$ for some $p \in T^{2}$. Thus in the prime orientable case, we need only analyze the prime $\omega$-harmonic maps $f: T^{2} \rightarrow M$ which fail to be somewhere injective.

Following the proof of Theorem 3 in [Sampson 1978], we note that it follows from Aronsjazn's unique continuation theorem for harmonic maps that if $d f$ has rank zero on a nonempty open set, the harmonic map $f$ must be constant. If $d f$ has rank one on a nonempty open set $U \subset \Sigma$, every point of $U$ has an open neighborhood which is mapped by $f$ onto a smooth $\operatorname{arc} C$ in $M$. We can suppose that coordinates $(u, \theta)$ have been constructed on $U$ so that $\partial f / \partial \theta=0$, and thus $f: U \rightarrow M$ reduces to a function of one variable, $f(u, \theta)=f_{0}(u)$, parametrizing a curve $C$. In this case the harmonic map must be a parametrization of a geodesic.

Finally, we need to analyze the case in which $d f$ has rank two on an open set. This includes harmonic maps with fold points at which the rank drops to one. 
To analyze such maps, we let

$$
F=\{p \in \Sigma: L(p)=\overline{\boldsymbol{L}}(p)\},
$$

points at which the rank of $d f$ is one, and carry through the theory of branched immersions described in [Gulliver et al. 1973], allowing now, however, for the possibility of folding of $f$ along $F$.

We can carry out the analysis for an arbitrary Riemann surface, not just a torus or Klein bottle. We begin by defining an equivalence relation $\sim$ on points of $\Sigma-$ $F$ by setting $p \sim q$ if there is are open neighborhoods $U_{p}$ and $U_{q}$ of $p$ and $q$ respectively, and a conformal or anticonformal diffeomorphism $\psi: U_{p} \rightarrow U_{q}$ such that $f \circ \psi=f$. Using the argument in [Gulliver et al. 1973], which is based upon Aronsjazn's unique continuation theorem, one shows that $\sim$ is indeed an equivalence relation and that the quotient space $\Sigma_{0}=\Sigma-F / \sim$ is a smooth manifold except at branch points if they exist. Moreover, the conformal structure $\omega$ on $\Sigma$ projects to a conformal structure $\omega_{0}$ on $\Sigma_{0}$. (In the case where $\Sigma$ is a torus and $f$ is not conformal, $f$ has no branch points, of course.) We can define $f_{0}: \Sigma_{0} \rightarrow M$ by $f_{0}([p])=f(p)$, where $[p]$ denotes the equivalence class of $p$, so that if $\pi: \Sigma \rightarrow \Sigma_{0}$ is the quotient map, $f_{0} \circ \pi=f$. We note that any point equivalent to a branch point is itself a branch point. The restriction of $f_{0}$ to $\Sigma_{0}$ minus the equivalence classes of the branch points is a harmonic map of finite energy. It therefore follows from the removable singularity theorem of Sacks and Uhlenbeck [1981, Theorem 3.6] that the restriction of $f_{0}$ can be extended to the equivalence classes of the branch points so as to be a harmonic map.

If $f$ has fold points, $\Sigma_{0}$ will consist of several connected components (at least two). Each such component will be diffeomorphic to a component of $\Sigma-F$. Moreover, two components of $\Sigma-F$ which have the same components of $F$ in their closure must be diffeomorphic. In the case of the torus this implies that no component of the set $F$ of fold points can be a null homotopic circle, because this would imply that a disk is diffeomorphic to something which is not a disk. In the case where $\Sigma$ is a torus, all of the components of $F$ must be smooth closed circles and must all lie in the same homology class.

Note that the complex dilatation $K_{f}(p)$ as described in [Bers 1960] or [Imayoshi and Taniguchi 1992] must go to infinity at points of $F$. Moreover, $K_{f_{0}}([p])=$ $K_{f}(p)$. It follows that all components of $\Sigma-F$ are diffeomorphic to each other. Moreover, if there were more than two components, then $f$ would not be prime, and hence there are exactly two sheets to the covering $\pi: \Sigma-F \rightarrow \Sigma_{0}$.

We can define a map $B: \Sigma \rightarrow \Sigma$ which fixes $F$ and interchanges the two sheets of the cover; thus $f \circ B=f$. The map $B$ is an orientation-reversing isometry of $T^{2}$ with its flat metric, and it induces a map (also denoted by $B$ from $\boldsymbol{L}$ to $\overline{\boldsymbol{L}}$ such that $B \circ \pi=\pi \circ B$, where $\pi: L \rightarrow \Sigma$ is the projection. 
The isometry $B: T^{2} \rightarrow T^{2}$ induces a conjugate linear involution

$$
B_{*}: \boldsymbol{E} \rightarrow \boldsymbol{E}, \quad \text { where } \quad \boldsymbol{E}=f^{*} T M \otimes \mathbb{C},
$$

and we divide the space $\Gamma(\boldsymbol{E})$ of sections of $\boldsymbol{E}$ into a direct sum $\Gamma(\boldsymbol{E})=\Gamma_{+}(\boldsymbol{E})+$ $\Gamma_{-}(\boldsymbol{E})$, where

$$
\Gamma_{+}(\boldsymbol{E})=\left\{X \in \Gamma(\boldsymbol{E}): B_{\star}(X)=\bar{X}\right\}, \quad \Gamma_{-}(\boldsymbol{E})=\left\{X \in \Gamma(\boldsymbol{E}): B_{\star}(X)=-\bar{X}\right\} .
$$

The sections of $\Gamma_{+}(\boldsymbol{E})$ can be seen as deformations of the harmonic cylindrical band, while the sections of $\Gamma_{-}(\boldsymbol{E})$ are deformations of $f$ that separate the two sheets of $\Sigma_{0}$.

We can now apply the argument of Section 4 to $f$. Once again, we see that variations in the metric on $M$ eliminate all of the potential Jacobi fields for the function $E_{\omega}$. Thus an application of the Sard-Smale theorem [Smale 1965] shows that for generic choice of metric there are no Jacobi fields tangent to the surface except for the those generated by the action of the symmetry group $S^{1} \times S^{1}$.

The argument for the case of a prime harmonic Klein bottle $f: K^{2} \rightarrow M$ is similar. in this case, $f$ is double covered by a harmonic torus $\tilde{f}: T^{2} \rightarrow M$ with covering transformation $A$. If $f$ is not somewhere injective, we construct $\Sigma_{0}$ as above, but now $\Sigma_{0}$ has only one component and the isometry $B: K^{2} \rightarrow K^{2}$ fixing $F$ lifts to an isometry $B: T^{2} \rightarrow T^{2}$ on the double cover which commutes with $A$. We can now divide the space $\Gamma(\boldsymbol{E})$ into a direct sum

$$
\Gamma(\boldsymbol{E})=\Gamma_{++}(\boldsymbol{E})+\Gamma_{+-}(\boldsymbol{E}) \oplus \Gamma_{-+}(\boldsymbol{E})+\Gamma_{--}(\boldsymbol{E}),
$$

where, for example,

$$
\Gamma_{+-}(\boldsymbol{E})=\left\{X \in \Gamma(\boldsymbol{E}): A_{\star}(X)=\bar{X}, B_{\star}(X)=-\bar{X}\right\} .
$$

the other summands being defined similarly. Once again, we apply the previous argument and the Sard-Smale theorem to finish the proof of Theorem 2.

\section{Acknowledgment}

The author takes pleasure in thanking the referee for helpful suggestions leading to an improvement in the article.

\section{References}

[Abraham 1970] R. Abraham, "Bumpy metrics", pp. 1-3 in Global analysis (Berkeley, 1968), vol. 1, edited by S.-S. Chern and S. Smale, Proc. Sympos. Pure Math. 14, Amer. Math. Soc., Providence, R.I., 1970. MR 42 \#6875 Zbl 0215.23301

[Bers 1960] L. Bers, "Quasiconformal mappings and Teichmüller's theorem”, pp. 89-119 in Analytic functions (Princeton, 1957), edited by R. Nevanlinna et al., Princeton Univ. Press, Princeton, N.J., 1960. MR 22 \#5716 Zbl 0100.28904 
[Bott 1956] R. Bott, "On the iteration of closed geodesics and the Sturm intersection theory", Comm. Pure Appl. Math. 9 (1956), 171-206. MR 19,859f Zbl 0074.17202

[Bott 1982] R. Bott, "Lectures on Morse theory, old and new", Bull. Amer. Math. Soc. (N.S.) 7:2 (1982), 331-358. MR 84m:58026a Zbl 0505.58001

[Gulliver et al. 1973] R. D. Gulliver, II, R. Osserman, and H. L. Royden, "A theory of branched immersions of surfaces", Amer. J. Math. 95 (1973), 750-812. MR 50 \#14595 Zbl 0295.53002

[Imayoshi and Taniguchi 1992] Y. Imayoshi and M. Taniguchi, An introduction to Teichmüller spaces, Springer, Tokyo, 1992. MR 94b:32031 Zbl 0754.30001

[Klingenberg 1978] W. Klingenberg, Lectures on closed geodesics, Grundlehren der Math. Wissenschaften 230, Springer, Berlin, 1978. MR 57 \#17563 Zbl 0397.58018

[Klingenberg and Takens 1972] W. Klingenberg and F. Takens, "Generic properties of geodesic flows", Math. Ann. 197 (1972), 323-334. MR 46 \#6402 Zbl 0225.58006

[Lawson 1980] H. B. Lawson, Lectures on minimal submanifolds, vol. 1, Mathematics lecture series 9, Publish or Perish, Berkeley, 1980. Zbl 0434.53006

[McDuff and Salamon 2004] D. McDuff and D. Salamon, J-holomorphic curves and symplectic topology, American Mathematical Society Colloquium Publications 52, American Mathematical Society, Providence, RI, 2004. MR 2004m:53154 Zbl 1064.53051

[Micallef and Moore 1988] M. J. Micallef and J. D. Moore, "Minimal two-spheres and the topology of manifolds with positive curvature on totally isotropic two-planes", Ann. of Math. (2) 127:1 (1988), 199-227. MR 89e:53088 Zbl 0661.53027

[Moore 2006] J. D. Moore, "Bumpy metrics and closed parametrized minimal surfaces in Riemannian manifolds", Trans. Amer. Math. Soc. 358:12 (2006), 5193-5256. Correction to appear. MR MR2238914 Zbl 05120558

[Sacks and Uhlenbeck 1981] J. Sacks and K. Uhlenbeck, "The existence of minimal immersions of 2-spheres”, Ann. of Math. (2) 113:1 (1981), 1-24. MR 82f:58035 Zbl 0462.58014

[Sacks and Uhlenbeck 1982] J. Sacks and K. Uhlenbeck, "Minimal immersions of closed Riemann surfaces”, Trans. Amer. Math. Soc. 271:2 (1982), 639-652. MR 83i:58030 Zbl 0527.58008

[Sampson 1978] J. H. Sampson, "Some properties and applications of harmonic mappings", Ann. Sci. École Norm. Sup. (4) 11:2 (1978), 211-228. MR 80b:58031 Zbl 0392.31009

[Simons 1968] J. Simons, "Minimal varieties in riemannian manifolds", Ann. of Math. (2) 88 (1968), 62-105. MR 38 \#1617 Zbl 0181.49702

[Smale 1965] S. Smale, "An infinite dimensional version of Sard's theorem", Amer. J. Math. 87 (1965), 861-866. MR 32 \#3067 Zbl 0143.35301

[Wolf 1967] J. A. Wolf, Spaces of constant curvature, McGraw-Hill, New York, 1967. MR 36 \#829 Zbl 0162.53304

Received August 17, 2005. Revised February 13, 2007.

JoHn Douglas MoORE

DEPARTMENT OF MATHEMATICS

UNIVERSITY OF CALIFORNIA

SANTA BARBARA, CA 93106

UNITED STATES

moore@math.ucsb.edu

http://www.math.ucsb.edu/ moore 